\title{
Assessment of Macro Fiber Composite Sensors for Measurement of Acoustic Partial Discharge Signals in Power Transformers
}

\author{
Bruno Albuquerque de Castro, Member, IEEE, Danilo de Melo Brunini, Member, IEEE, \\ Fabricio Guimarães Baptista, Member, IEEE, André Luiz Andreoli, Member, IEEE, \\ and José Alfredo Covolan Ulson
}

\begin{abstract}
This paper presents a performance assessment of macro fiber composite (MFC) sensors for measuring acoustic emission (AE) signals from partial discharges (PD) in power transformers filled with mineral oil. MFC sensors are low-profile and flexible, allowing them to be attached to uneven surfaces, such as a transformer wall. Two types of MFC sensors were assessed: P1 (d33 effect) and P2 (d31 effect), which are optimized for different deformations in the structure, such as elongation and contraction, respectively. In addition, a conventional AE sensor, R15I-AST model from Physical Acoustics South America, was also used as a reference for comparative analysis. Four metrics were applied to the signals: root mean square, energy criterion, Akaike criterion, power spectral density, and correlation. The experimental results indicate a high similarity between the MFC sensors and the conventional $\mathrm{AE}$ sensor, which expands the research field in acoustic $P D$ measurement in power transformers by using low-cost and flexible sensors.
\end{abstract}

Index Terms-MFC Sensor, acoustic emission, partial discharges, transformers, low cost system.

\section{INTRODUCTION}

$\mathbf{P}$ OWER Transformers are high-cost equipment and essential for the electrical system operation. During the operation of power transformers, some factors such as overload, nonlinear loads and switching can cause the degradation of the insulating system, which allows the occurrence of internal partial discharges [1]-[3]. Partial discharges (PD) are random phenomena which have a direct influence on the transformers failures as well as on the interruptions in the power system. In this context, PD identification systems have been established as useful tools to support corrective maintenance in power transformers and contributed to reduce the costs involved in this task. Online PD monitoring can reduce the severe failures due to damage caused in the insulation system [2]-[8].

Due to the need of PD monitoring in transformers, several methods were developed such as electrical, optical and

Manuscript received July 9, 2017; accepted July 27, 2017. Date of publication August 3, 2017; date of current version August 22, 2017. This work was supported by the São Paulo Research Foundation under Grant 2015/02500-6 and Grant 2015/24903-5. The associate editor coordinating the review of this paper and approving it for publication was Prof. Bernhard Jakoby. (Corresponding author: Bruno Albuquerque de Castro.)

The authors are with the São Paulo State University (UNESP), School of Engineering, Bauru, Department of Electrical Engineering, 17033-360 BauruSP, Brazil (e-mail: bruno.castro@feb.unesp.br; danilo.brunini@ieee.org; fabriciogb@feb.unesp.br; andreoli@feb.unesp.br; ulson@feb.unesp.br).

Digital Object Identifier 10.1109/JSEN.2017.2735858 electromagnetic measurements, chemical analysis and acoustic emission (AE) [9]-[13]. A complete measurement system can combine two of these methods for the proper detection and localization of the PD. As is well known, the electromagnetic method has high sensitivity and it can be applied in online monitoring for PD detection [12]. In contrast, the main advantage of the AE method is the ability to determine the localization of the PD source [3], [13]. Typically, this principle uses several AE sensors installed on the transformer wall to detect ultrasound waves generated by the PD and the localization of the PD-source is determined based on the time of flight differences between the acoustic PD signals [10]-[14].

The use of two combined measurement methods is required because the signals from the AE sensors have low signal-tonoise ratio (SNR) due to the damping that the acoustic waves undergo in the propagation paths between the PD-source and the sensors [14], [15]. Therefore, the measurement of the electromagnetic signals in the ultra-high frequency (UHF) range, which has high sensitivity, is typically used for the PD detection and the triggering of the $\mathrm{AE}$ measurement to reduce noise by the averaging of the acoustic signals, allowing the reliable localization of the PD-source [12], [14], [16].

In the present study, we compared the performance of alternative sensors for the measurement of AE signals aiming the PD localization. Although there are many types of consolidated AE sensors for industrial and academic applications, these sensors have high financial cost, limiting the use of this technique for the failure diagnosis in transformers. Therefore, the use of low-cost alternative sensors is attractive for the PD localization, especially in large transformers where several sensors may be required to determine the PD-source accurately.

A recent study [3] has proposed the use of piezoelectric diaphragms, commonly known as buzzers, for measuring acoustic PD signals in power transformers. These components are readily available at very low cost and the results indicate their viability to detect AE signals from PD events. However, similarly to the conventional AE sensors, the diaphragms are rigid and little flexible, which does not allow its attachment to uneven surfaces, such as transformers made with the cylindrical shape or external parts in this form. For these cases, rigid sensors can not be used because their surfaces not adhere fully to curved structures. In order to overcome this drawback, 
in this study we present an experimental evaluation of macro fiber composite (MFC) sensors for acoustic PD measurement in power transformers. These devices are low-profile, durable and flexible, allowing their attachment to uneven surfaces, which would not be feasible with common sensors. The MFCs were first developed by NASA in 1996 and recently used in many applications such as sensors, actuators and energy harvesting [17], [18].

In this context, the objective of this paper is to present an experimental analysis of the feasibility of using MFC sensors to detect PD in power transforms based on the AE method. Two types of sensors have been used: P1 (d33 effect) and P2 (d31 effect), as detailed in Section III. Both sensors were connected at the external wall of a transformer to measure AE signals from PD events. To verify the feasibility of the proposed sensors, the signals obtained for the both MFCs were compared with a R15I-AST sensor from Physical Acoustics South America - PASA®, which is a conventional sensor consolidated in academic and industrial applications for acoustic PD measurement.

It is worth mentioning that in this study a full discharge was generated instead of a PD, according to the experimental setup presented in Section IV. Although the amplitudes of the acoustic signals generated by a full discharge are higher compared to a realistic PD, the results presented in this study are valid because the sensors were analyzed and compared under identical conditions. The feasibility of the MFC sensors was assessed and compared to the conventional AE sensor by the analysis of time-domain and frequency-domain responses when they were subjected to the same full discharge.

The results show that the MFC sensors and the conventional AE sensor have similar behaviors for acoustic PD measurement. Base on in this similarity, the proposed P1 and P2 MFC sensors can be a real alternative that contributes to the development of PD localization systems and improvement in the quality of electricity supply or planning maintenances.

The remainder of this paper is organized as follows. Section II presents the basic theory of PD, Section III talks about MFC piezoelectric sensors, Section IV presents the materials and method, Section $\mathrm{V}$ discuss de results and finally the conclusions are presented in Section VI.

\section{Acoustic Partial Discharges Measurement IN POWER TRANSFORMERS}

There is a great concern about the operating integrity during the life-cycle of power transformers because these devices are the most expensive and critical equipment in electric power plants. Therefore, there are a significant importance in monitoring the insulation condition of power transformers.

The insulation deterioration starts with some factors such as overload, transient voltages by switching and atmospheric origin, nonlinear loads, which degrade the insulation material. These factors create the ideal environment for the incidence of PDs, which may completely compromise the useful life of the transformer [1]-[5]. One of the main methods to evaluate the insulation integrity of an electrical equipment is by monitoring the PD, which is defined by the IEC 60270 as "localized electrical discharges that only partially bridges the insulation between conductors" [9].

An electric field incident on the dielectric material makes enough changes that produce an electric break, arising a current in a specific area closing partially the circuit. Generally, these materials are degraded or have voids that promoting a material discontinuity. The voids cause electrical field enhancement and so originate $\mathrm{PD}$, creating a load pulse lasting less than $1 \mu$ s that provides a destructive energy releasing heat, light, electromagnetic radiation, pressure ultrasound waves that radiate in all directions from a source [10], [11], [19]-[22].

In the power transformer when the PD occurs, the longitudinal waves travel thought mineral oil and refract in the surfaces of the steel tank, yielding longitudinal and share waves. Accordingly, these refracted waves in the steel surfaces can be detected using AE sensors attached to the steel wall [23]. Therefore, this paper presents an experimental analysis of the feasibility of MFC sensors for acoustic PD measurement, in order to enable the large-scale use of this noninvasive method of PD localization. The piezoelectric effect and the MFC sensors are presented in the next section.

\section{Piezoelectricity And MFC Sensors}

The property that some dielectrics have to create a polarization when subjected to a mechanical stress is called piezoelectricity. This polarization produced by the tensile stress produces a voltage output through the formation of an electric dipole in material [24]. The reverse effect also occurs: a mechanical deformation arises applying an electrical voltage between the two sides of the piezoelectric material.

In piezoelectricity, the quantities involved are tensors, despite some particular directions of the crystals, the vectors are in the same direction [24]. The relations of scalar quantities are given by (1) and (1), respectively:

$$
\begin{aligned}
& D_{i}=d_{i k l} T_{k l}+\varepsilon_{i k}^{T} E_{k} \\
& S_{i j}=s_{i j k l}^{E} T_{k l}+d_{k i j} E_{k}
\end{aligned}
$$

where $E_{k}$ is the electric field component, $s_{i j k l}^{E}$ is the elastic compliance constant at constant electric field, $\varepsilon_{i k}^{T}$ is the permittivity component at constant stress, $d_{i k l}$ and $d_{k i j}$ are the piezoelectric constants, $D_{i}$ is the electric displacement component, $S_{i j}$ is the strain component, $T_{k l}$ is the traction vector component. The subscripts $i, j, k$ and $l$ take the values 1,2 and 3, and represent the natural coordinate system of the piezoelectric material.

According to (1) and (2), there is an electric load owing to a mechanical stress and a deformation due to an electrical field. Therefore, in piezoelectric sensors there is an electromechanical coupling, allowing to generate a voltage when excited by mechanical deformations caused by an acoustic PD wave.

Normally, the conventional AE sensors have a high average cost, such as R15I, which is consolidated for AE applications [25]. This sensor is enclosed in a metal housing and incorporates a low-noise input preamplifier and a filter all inside the house, as shown in Fig. 1 (a).

The alternative piezoelectric sensors used in this study were the MFCs sensors [17], [26], which are low-profile, durable 


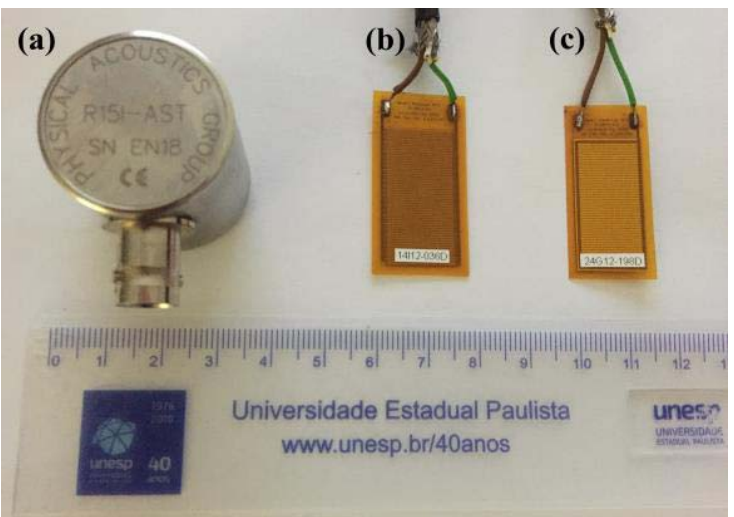

Fig. 1. Sensors used in this study: (a) R15I-AST conventional AE sensor, (b) MFC-P1 and (c) MFC-P2 sensor.

and flexible devices composed of rectangular piezoceramic rods inserted between layers of adhesive, electrodes and polyimide film as shown Fig. 1 (b) and (c). The electrodes are attached to the film in an interdigitated pattern, which transfer the applied voltage directly to and from the ribbon shaped rods.

These devices are as a thin, surface-conformable sheet and can be applied to various types of structures or embedded in composite structures [17], [26].

The MFC sensors used in this research are the M2814-P1 and M2814-P2 from Smart Material Corporation, Sarasota, FL, USA. The first type (P1) uses d33 effect in which the piezoelectric material is only polarized longitudinally with respect to its plane. This effect is related to the elongation of the structure. The nominal capacitance of this sensor is $1.15 \mathrm{nF}$. For the second type (P2), the polarization is perpendicular with respect to its plane, which is related to the contraction of the structure, and its nominal capacitance is $30.78 \mathrm{nF}$. Therefore, the MFC-P1 has a higher capacitive reactance than MFC-P2.

Several low-cost piezoelectric sensors such as lead zirconate titanate (PZT) ceramics and piezoelectric diaphragms (buzzers) has been applied in many scientific studies for structural health monitoring (SHM) [27]-[33] and PD detection [3] in power transforms, and good results have been reported. Therefore, as a proposal, this study presents an experimental analysis of the feasibility of P1 and P2 MFC sensors for acoustic PD measurement. As mentioned before, these devices are flexible, allowing its attachment to many uneven surfaces of the transformer which would not be accessible with conventional sensors. The experimental procedure is presented in the next section.

\section{EXPERIMENTAL SETUP}

Since the purpose of this research was to realize a comparative analysis of the performance between the conventional $\mathrm{AE}$ sensor and the flexible MFC sensors for acoustic PD measurement, it was necessary to generate a discharge in a transformer equipped with these sensors. However, instead of a real PD, a full discharge was generated.

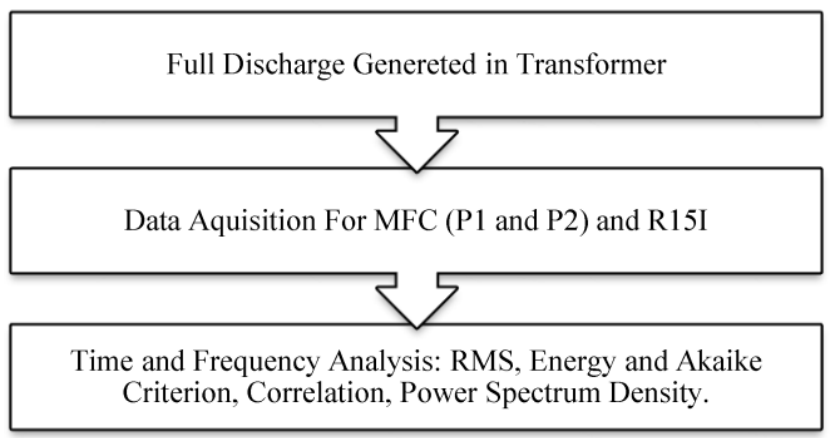

Fig. 2. Signal analysis flowchart.

A full discharge was used in this study because normally the propagation paths of the acoustic waves between a real PD-source and the AE sensors attenuate the acoustic signals, making the SNR of the acoustic measurements low and requiring an additional method such as the electromagnetic for triggering, as mentioned before [12], [14], [16]. In contrast, a full discharge allows acoustic measurements with high SNR, requiring no additional methods for the effective analysis and comparison of the sensors, which is the purpose of this study.

Although a full discharge was applied in the power transformer, this approach is valid because the three sensors were analyzed and compared under the same conditions. In addition, according to the results presented in Section V, the MFC sensors show a time-domain and frequency-domain responses very similar to the conventional $\mathrm{AE}$ sensor, indicating the feasibility of these sensors for the localization of real PDs.

The flowchart followed to perform the analysis and comparison of the three sensors is shown in Fig. 2. Each of the steps shown in the flowchart of Fig. 2 are detailed in the following sections.

\section{A. Assembling and PD Data Acquisition}

The experimental configuration diagram is shown in Fig. 3 and the transformer with the conventional $\mathrm{AE}$ sensor and the two types of MFC sensors are shown in Fig. 4. The three sensors were fastened in a $30 \mathrm{kVA}$ transformer's wall using liquid paraffin. An instrumentation amplifier (INA $128 \mathrm{P}$, Texas Instruments) with frequency response up to $500 \mathrm{kHz}$ and gain of $28 \mathrm{~dB}$ was used to amplifier the signals from the P1 and P2 MFC sensors. Furthermore, this amplifier was also used as an antialiasing filter. To avoid electromagnetic disturbances in the sensors, the wall of transformer and the cables shield has been grounded to conduct only AE signals related to the discharges. In addition, a high-pass filter with $20 \mathrm{kHz}$ cutoff frequency was used to eliminate low frequency vibrations.

Once the full discharge was generated, the data acquisition was performed using an oscilloscope with sample rating set to $10 \mathrm{MS} / \mathrm{s}$.

The discharges were generated in the insulation oil of the transformer applying a $7 \mathrm{kVac}$ voltage in a copper electrode as shown in Fig. 3. To generate this voltage, 100 Vac was applied in a potential transformer whose transform relation is 1:70. 


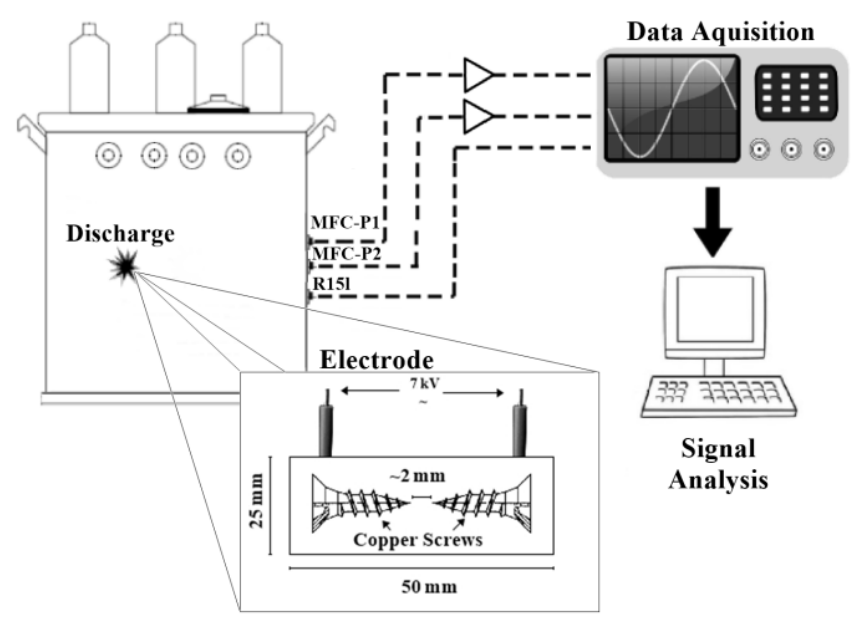

Fig. 3. Schematic setup.

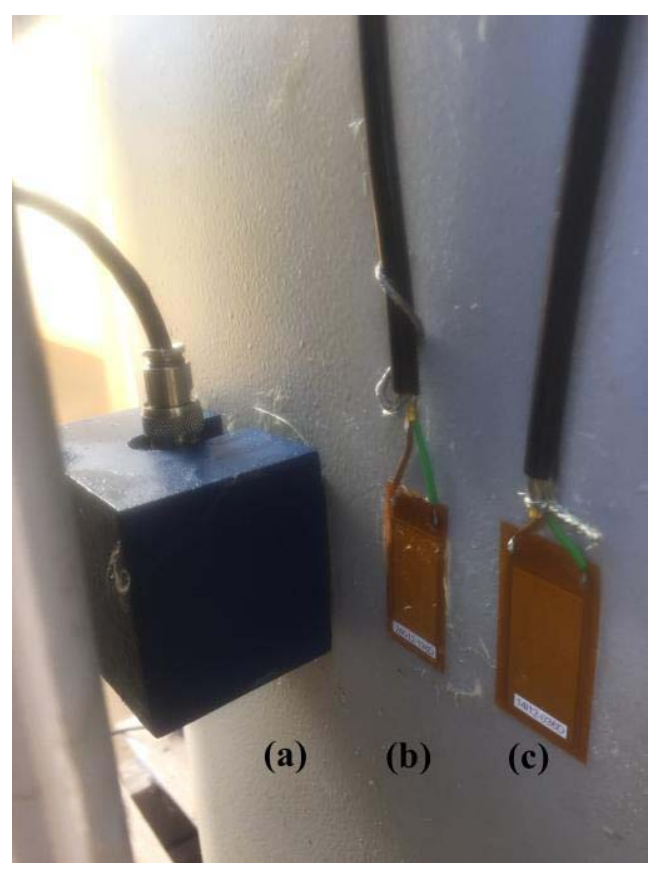

Fig. 4. Sensors installed on the transformer wall: (a) R15I-AST conventional AE sensor, (b) MFC-P2 and (c) MFC-P1 sensor.

This point to point electrode was made with cooper screws attached to an insulation material. A point-to-point gap was used in this study because the PD in the point-to-point system in oil can be related to PDs generated by insulation damage of two neighboring turns of winding of a transformer [34].

\section{B. Signal Processing Parameters}

The AE data provided by the oscilloscope allowed performing analysis in the time and frequency domain. Several routines were developed in Matlab for time and frequency domain analysis, such as energy criterion, root mean square (RMS), Akaike criterion and power spectral density (PSD) method. In the time domain analysis, metrics were associated with basic statistics analysis such as correlation and linear fit. The conventional sensor (R15I) was the reference for this work.
1) Energy Criterion: The purpose of energy criterion is to characterize signals which frequency range and energy content have variation, as acoustic emission waves [13], [35]. This criterion enables the detection of the acoustic wave arrivals time in the sensors, as well as changes in the signal over time from a sophisticated algorithm. The energy curve $S(k)$ of the sampled signal $x$ is defined as the cumulative sum of amplitude values as shown in (3)

$$
S(k)=\sum_{k=1}^{i}\left(x_{k}^{2}-\frac{i . S_{N}}{N}\right)
$$

where $i$ is a count variable or part of the signal, and the instantaneous energy curve depends on the total energy of the $S_{N}$ signal and the number of samples $N$.

The global minimum of this curve corresponds to the start time of the AE signal for the sensor, i.e., the time at which a sensor is excited initially by ultrasound waves. The ultrasound waves excite the AE sensors by waves reflections, refractions and directly. For PD location is very important know the time of flight differences between acoustic PD signals. Using an average sound velocity mainly geared to the propagation speed in oil only, signal portions of the direct way should be denoted as 'PD-signal' and the structure-borne interferences can be addressed as 'PD-Noise' instead [13].

According to the results shown in [13], the global minimum for the energy criterion could be used to localize the start time of the signals from the sensors because the energy criterion is a statistical method that compares the importance of each sample in the energy across the mean of the whole signal, minimizing the 'PD-Noise' effects. The signal energy intensity is directly related to the load applied to the sensor. Therefore, this criterion can be used as a way of characterizing and measuring a signal [13], [35].

2) Root Mean Square (RMS): RMS is one of the most important criteria to evaluating the signal acquired by the AE sensor [36]. This approach can be considered as a physical quantity of sound intensity and it is directly related to the load applied to the sensor, which makes it a very interesting value to be monitored. The RMS value of a finite time signal can be expressed by (4):

$$
E_{R M S}=\sqrt{\frac{1}{N} \sum_{k=1}^{N} x_{i}^{2}}
$$

where $N$ is the discrete number of samples equivalent to the interval of $1 \mathrm{~ms}, x_{i}$ is the sampled signal voltage of the sample $i$.

3) Akaike Information Criterion (AIC): The Akaike information criterion (AIC) considers the signals as autoregressive processes in which every sample is a linear combination of past values [13], [35].

This criterion is a measure of the goodness fit of a statistical model to a set of observations. It is commonly applied in seismology using a combination with two autoregressive fits to determine the onset of seismic waves by means of the local minimum in the AIC curve. In this paper, AIC is used to determine the time of arrival (TOA) of the signals and besides compare the performance of the conventional and MFCs curves. The model is performed by weighted signal 
variance $\sigma^{2}$ comparing from sample 1 to $n$ and $n+1$ to $N$, as shown in (5) [13], [35].

$$
A I C(k)=k \ln \left[\sigma^{2}(1, k)\right]+(N-k-1) \ln \left[\sigma^{2}(k+1, N)\right]
$$

4) Correlation Coefficient $(C c)$ : The correlation coefficient in (6) is calculated using one reference signal parameter, which is the signal from the R15I sensor $\left(x_{R S}\right)$, to perform the comparison with the signals from MFC sensors $\left(x_{P}\right)$, showing the similarity degree between them. Therefore, this study uses this method to assess the P1 and P2 MFC sensors for PD detection.

$$
C c=\sum_{k=0}^{n} \frac{\sum_{k=1}^{N}\left(x_{R S}(k)-\bar{x}_{R S}\right)-\left(x_{P}(k)-\bar{x}_{P}\right)}{\sqrt{\sum_{k=1}^{N}\left(x_{R S}(k)-\bar{x}_{R S}\right)^{2}}-\sqrt{\sum_{k=1}^{N}\left(x_{R S}(k)-\bar{x}_{P}\right)^{2}}}
$$

where $\bar{x}_{R S}$ and $\bar{x}_{P}$ are the mean values.

5) Power Spectral Density (PSD): PSD function is a standard method for the feature extraction of stochastic signals which describes the power distribution of a signal in the frequency domain [37], [38]. For estimation of this distribution, some parametric and non-parametric techniques are applicable. A typical non-parametric method is the Welch's periodogram [38]. In this method, a signal is divided into $L$ equal (possibly overlapping) segments whose length is $M$. The PSD of segment $i$ is given by (7):

$$
\hat{S}(\omega)^{i}=\frac{1}{\sum_{n=0}^{M-1} d_{M}^{2}(n)}\left|\sum_{n=0}^{M-1} x^{i}(n) d_{M} e^{-j \omega n}\right|^{2}
$$

where $d_{M}(n)$ is the windows function of length $M, x^{i}(n)$ is the $i_{t h}$ segment of $x(n)$ [37]. The average of $\hat{S}(\omega)^{i}$ of all $L$ segments is the PSD of $x(n)$ as shown in (8):

$$
\bar{S}_{x}(\omega)=\frac{1}{L} \sum_{i=1}^{L} \hat{S}(\omega)^{i}
$$

The signal processing parameters presented above were applied to the signals from the conventional $\mathrm{AE}$ sensor and MFC sensors for a comparative analysis of the feasibility for acoustic PD measurement. The results are presented and discussed in the next section.

\section{RESUlts AND Discussion}

In this section, the results of the comparative analysis of the signals from the three sensors in the time and the frequency domain are presented and discussed.

\section{A. Time-Domain Response}

The analysis of the time-domain response was performed by implementing the signal processing parameters described in the previous section in the Matlab software to compare the acoustic behavior of the three sensors used in this research, since the pure pure signal analysis is not enough to evaluate the feasibility of the MFC sensors to detect ultrasound waves. These parameters allow the features extraction of the acoustic content of the signals from the three sensors.
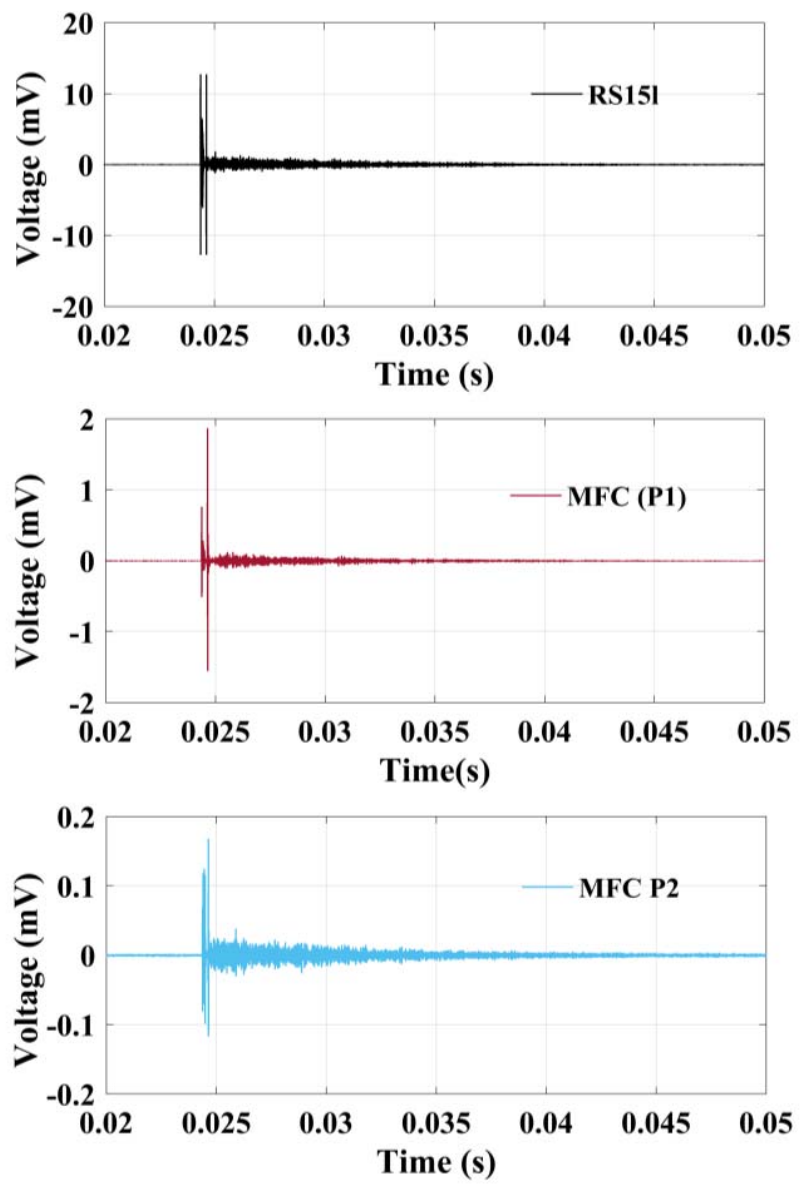

Fig. 5. AE signals obtained for the three sensors.

The discharge signals received by the R15I-AST, MFC-P1 and MFC-P2 are shown in Fig. 5.

There is a significant difference between the three signals in the time domain. The R15I-AST sensor gain is higher than the other two sensors. It was also observed that the MFC-P1 was more sensitivity than MFC-P2 to detection of AE signals. Perhaps, this result is mainly due to a difference capacitance of the both sensors. The MFC-P1 has a higher capacitive reactance than MFC-P2, according to explained in Section III. This causes a higher output voltage on the $\mathrm{P} 1$ sensor.

Although there are differences in the signal amplitudes, the signals suddenly increase to approximately $0.024 \mathrm{~s}$ and decrease until approximately $0.035 \mathrm{~s}$ for the three sensors.

To perform the statistic metrics such as RMS, energy criterion and AIC, the signals were normalized because the signal amplitudes of the sensors are different. The results obtained using the energy criterion are shown in Fig. 6.

We can observe that the energy responses for the acoustic load intensity applied to the sensors were very similar. The global minimum points in the graph represent the arrival time of the excitation, which was $24 \mathrm{~ms}$ for the three sensors and the maximum point of the energy curve occurred in $34 \mathrm{~ms}$ for all sensors as well.

For a better analysis, the linear approximations and correlation coefficients were computed to compare the energy curves 


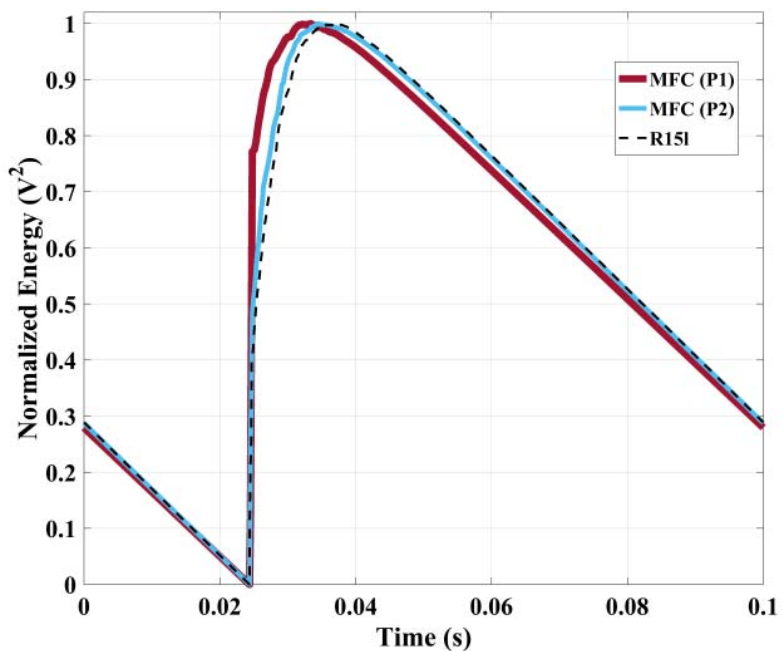

Fig. 6. Normalized energy criterion for the three sensors.

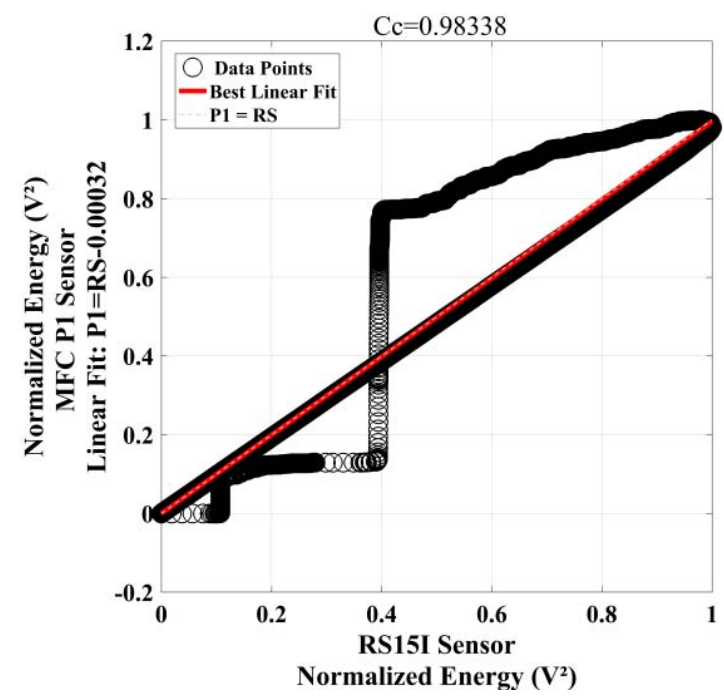

Fig. 7. Linear approximation and correlation coefficient for normalized energy signals, MFC P1 / R15I-AST.

of the three sensors. The purpose of this comparison is to show the acoustic excitation similarity between the MFC sensors and the conventional AE sensor. For the linear fit analysis, an angle close to 45 degrees indicates a high similarity between the signals. The linear approximations and correlation coefficients for the energy criterion obtained from the comparison of the P1 and P2 MFC sensors with the conventional AE sensor are shown in Fig. 7 and Fig. 8, respectively.

The correlation coefficient of MFC-P1 type sensor shown in Fig. 7 is 0.98338 which represents a high similarity between this sensor and the R15I-AST conventional sensor. The best linear fit observed is near to $45^{\circ}$, which also indicates that the ultrasound waves excited the sensors in very similar way.

The MFC-P2 analysis also indicated the good potential for acoustic PD measurement, since this sensor has high similarity with the conventional sensor, as shown in Fig. 8. In the comparison with the conventional sensor, the linear fit was nearly $45^{\circ}$ as well as MFC-P1 type and the correlation

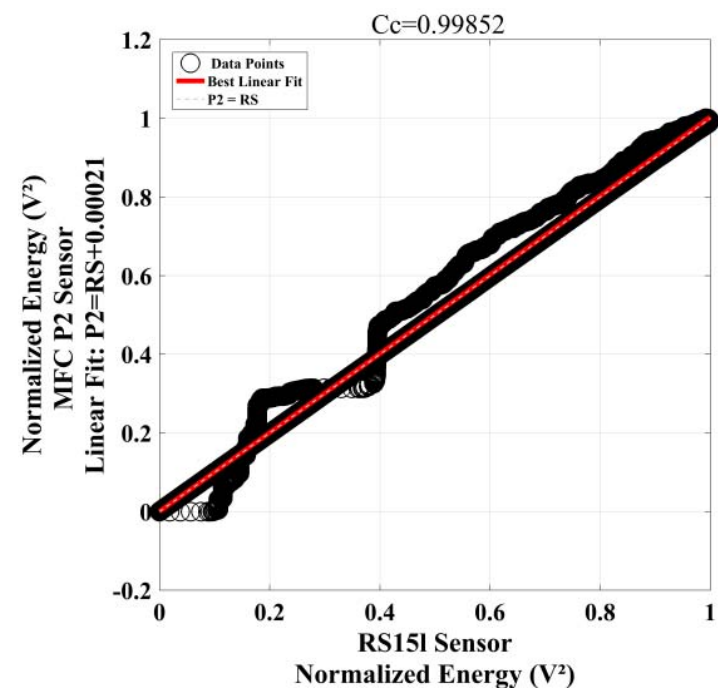

Fig. 8. Linear approximation and correlation coefficient for energy signals, MFC P2 / R15I-AST.
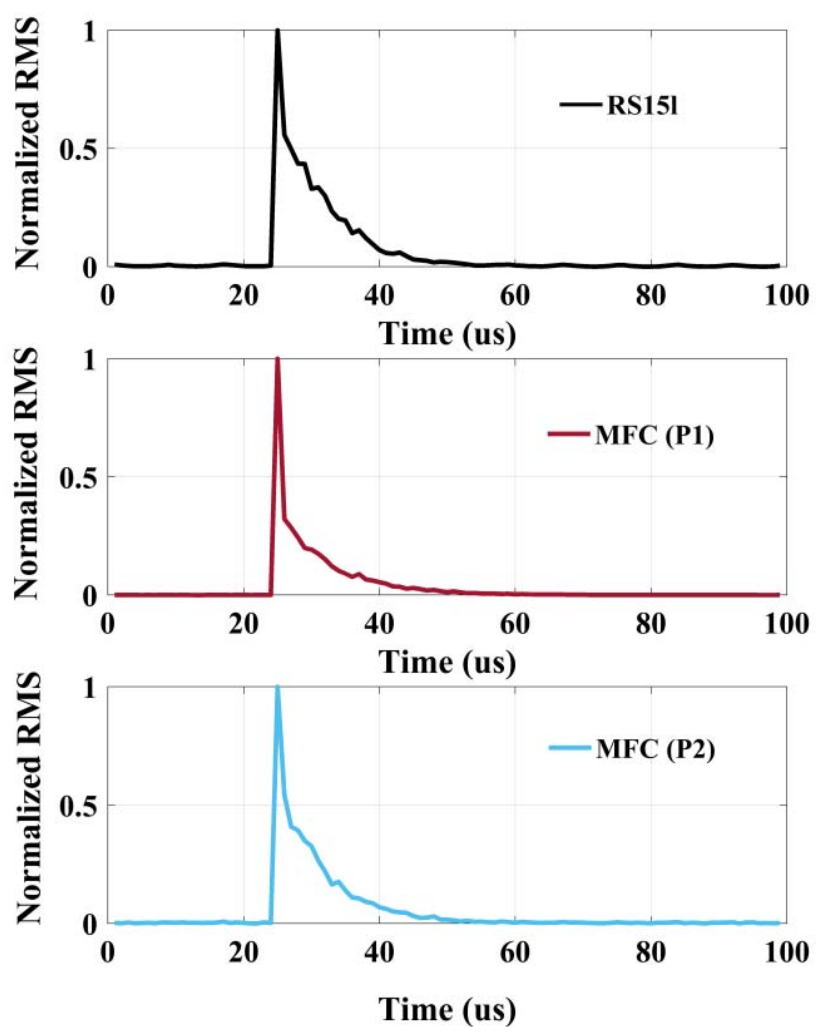

Fig. 9. Normalized RMS curves obtained for the three sensors.

coefficient was 0,99852. As noted, the two sensors proposed in this work obtained a similar behavior for the acoustic load from discharge.

Another criterion was performed by using the RMS analysis. The window period value considered was $1 \mathrm{~ms}$. Fig. 9 shows the RMS values for the conventional sensor, MFC-P1 and MFC-P2. This approach can be considered as a physical quantity of sound intensity and it is directly related to the load applied to the sensors, as well as the energy criterion. 
TABLE I

Time Values (Fig. 9)

\begin{tabular}{cccc}
\hline \hline RMS & \multicolumn{3}{c}{ Time $(\boldsymbol{\mu s})$} \\
\cline { 2 - 4 } $\begin{array}{c}\text { Value/Peak } \\
\text { Value (\%) }\end{array}$ & MFC P1 & MFC P2 & R15I-AST \\
\hline 100 & 25 & 25 & 25 \\
70.7 & 25.43 & 25.64 & 25.66 \\
10 & 34.25 & 37.41 & 38.77 \\
\hline \hline
\end{tabular}

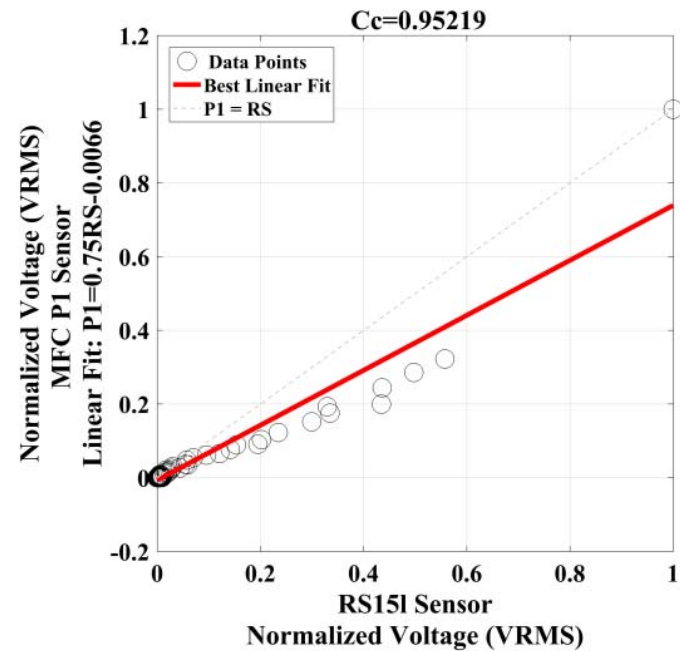

Fig. 10. Linear approximation and correlation coefficient for normalized RMS signals, MFC P1 / R15I-AST.

For each RMS curve, three points were highlighted to compare the signals as shown in Table I: the RMS peak value, an attenuation point with $70.7 \%$ of the peak ( $3 \mathrm{~dB}$ attenuation) and the last one, the attenuation point with $10 \%$ of the maximum RMS. These points were chosen for a quantitative comparison of the RMS curves and the intensity for acoustic emission load.

At time of $24 \mu \mathrm{s}$, the rate of change for the RMS signal was significant for all sensors and the peak was at $1 \mu$ s later. As the window was $1 \mathrm{~ms}$ in the RMS criterion, the time of arrival was $0.02 \mathrm{~s}$ as previously indicated by the energy criterion. Thus, the RMS analysis can be an alternative parameter to indicate the time of arrival signals. There is a significant similarity between the attenuation characteristics of the sensors, as it can be noticed in Fig. 9 and Table I. The $3 \mathrm{~dB}$ attenuation (70.7\% peak) occurred in $25.43 \mathrm{~ms}$ for the MFC-P1, $25.64 \mathrm{~ms}$ for MFC-P2 and $25.66 \mathrm{~ms}$ for the R15I-AST sensor. For the $10 \%$ attenuation of the peak value, the times were $34.25 \mathrm{~ms}$, $37.41 \mathrm{~ms}$ and $38.77 \mathrm{~ms}$ for MFC-P1, MFC-P2 and R15I-AST sensor, respectively.

Therefore, this criterion is a good tool for show the absolute values for contour waves. Although the discharge acoustic waves are impulsive, the damping of the acoustic emission waves can be observed with more details comparing to the original signal.

This similar behavior of the three sensors for discharge acoustic load is confirmed by computing the linear approximation and correlation coefficient. Fig. 10 and Fig. 11 show the results obtained from the comparison of the P1 and P2 MFC sensors with the conventional AE sensor, respectively.

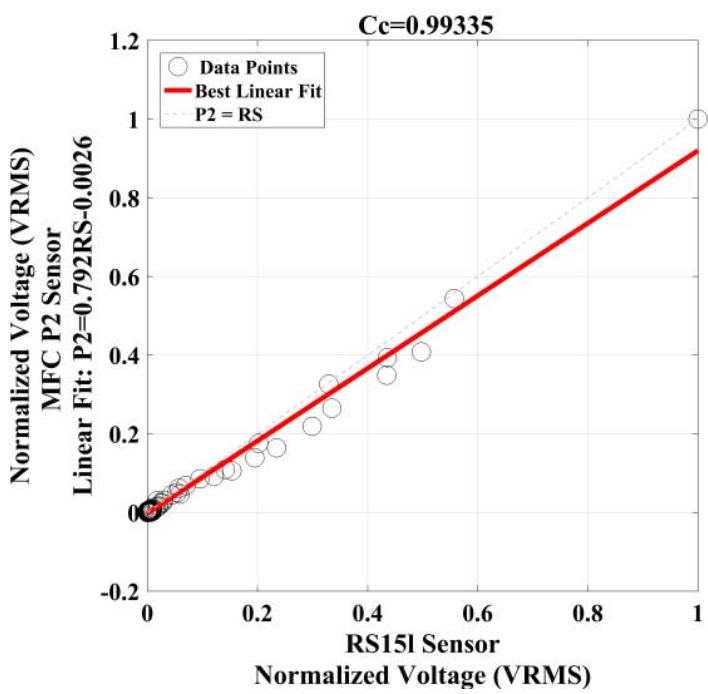

Fig. 11. Linear approximation and correlation coefficient for normalized RMS signals, MFC P2 / R15I-AST.

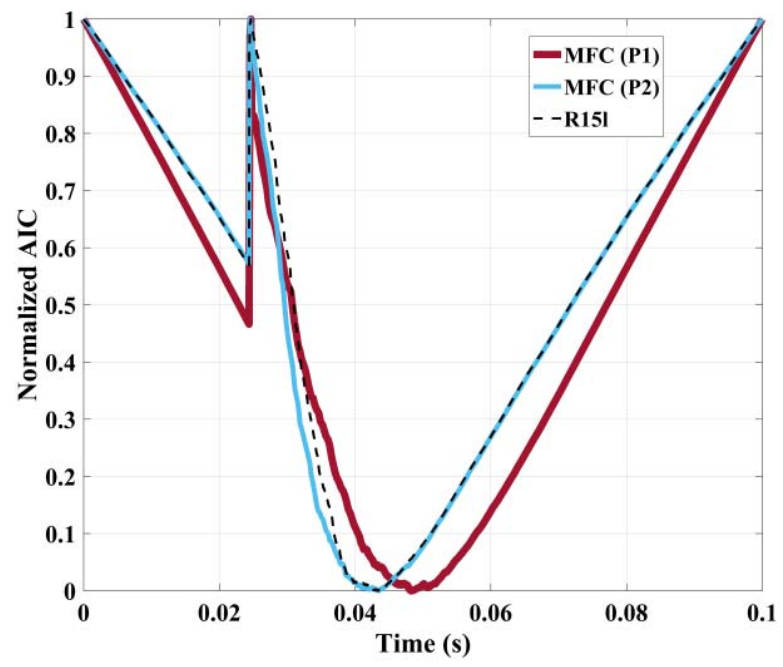

Fig. 12. Akaieke criterion obtained for the three sensors.

The correlation coefficient was 0.95219 for the MFC-P1 and 0.99335 for the MFC-P2 sensor. Although the linear fit was better to P2 sensor, there is a high degree of similarity between both sensors. Hence, the RMS criterion can contribute to the evaluation of similarity of acoustic signals, as indicated in the significant results obtained by the correlation and linear fit. In addition, this criterion also constitutes as a new approach to estimete acoustic emission time of arrival signals. For time of arrival signals in this method, it is necessary to convert the time scales due to use of window in the signal.

The AIC parameter was used as a measure of the goodness of fit for a statistical model to a set of observations of acoustic signals, indicating the high similarity between the signals from the three sensors. The results are shown in Fig. 12. According to Fig. 12, the curve decreases until a local minimum point that represents the time of arrival of the acoustic wave. For all sensors, this point was $0.24 \mathrm{~ms}$, showing that there is a consonance between the results showed by energy and RMS 


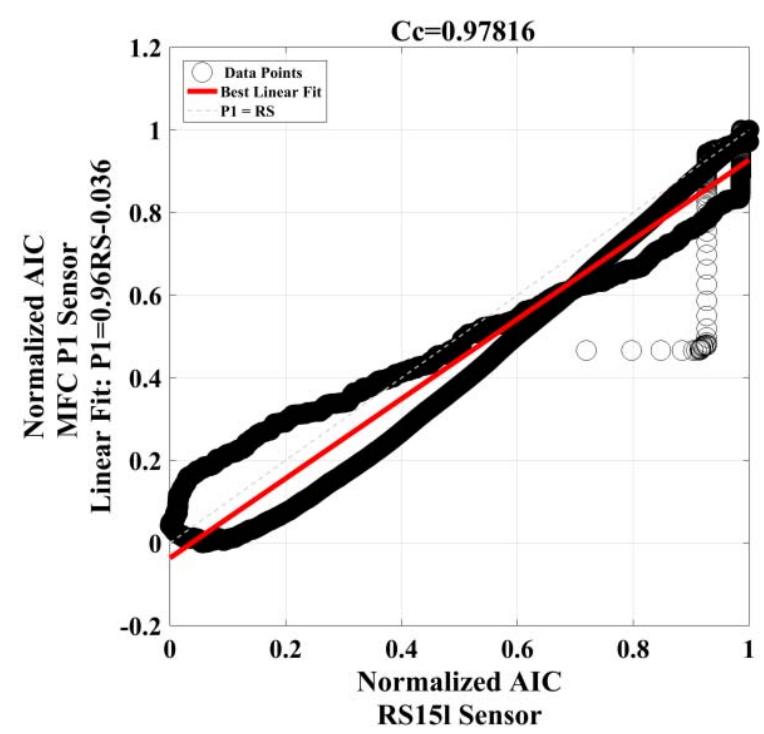

Fig. 13. Linear approximation and correlation coefficient for normalized Akaike Criterion, MFC P1 / R15I-AST.

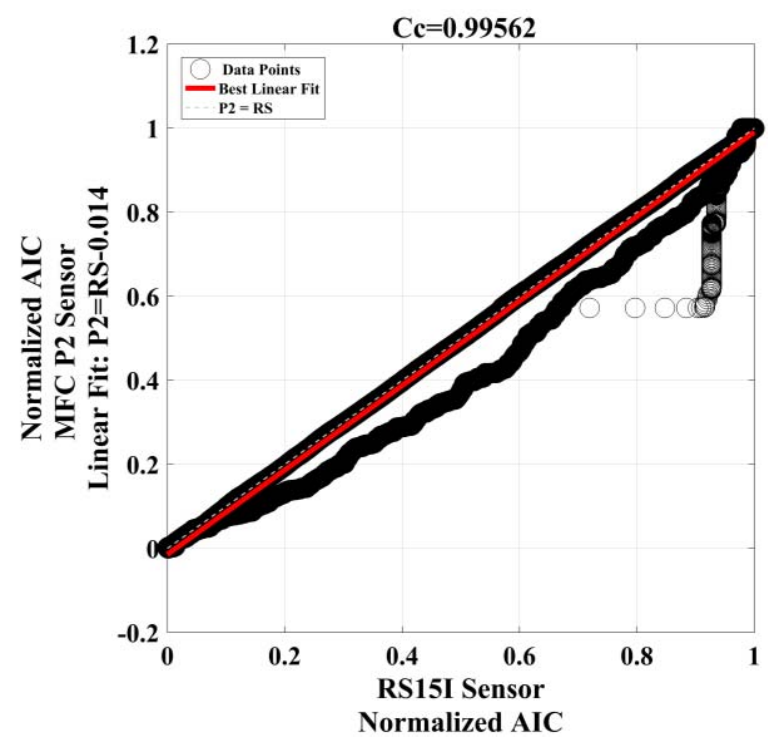

Fig. 14. Linear approximation and correlation coefficient for normalized Akaike Criterion, MFC P2 / R15I-AST.

criterion. Then, the AIC curve increases to a maximum point for all sensors.

Fig. 13 and Fig. 14 show the linear approximations and correlation coefficients for the AIC criterion obtained from the comparison of the P1 and P2 MFC sensors with the conventional AE sensor, respectively. The results indicate that the AIC curves obtained for the MFC-P1 and MFC-P2 sensor were, respectively, 0.9781 and 0.99562 similar to the conventional sensor. The linear fit was nearly $45^{\circ}$ for both sensors, considering as a reference the conventional sensor signal.

The analysis of the signals in the frequency domain are presented in the next section.

\section{B. Frequency-Domain Response}

The analysis of the PD signals in the frequency domain was performed computing the PSD as shown in Fig. 15.

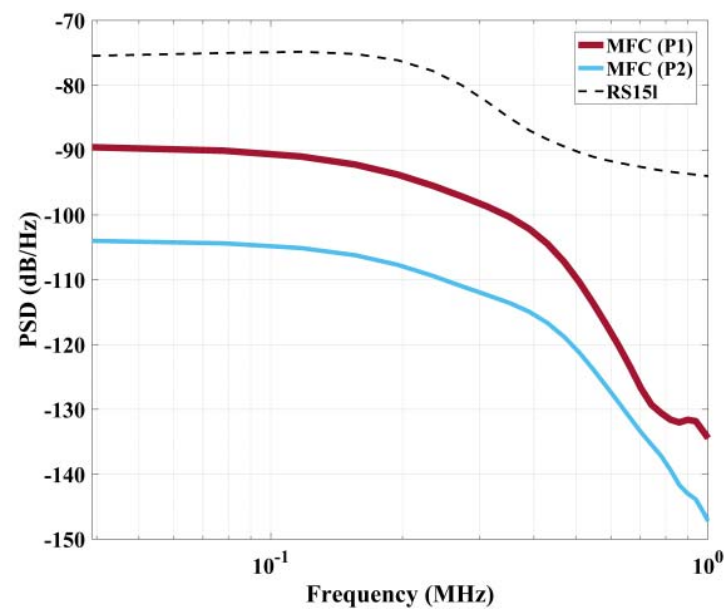

Fig. 15. PSD criterion applied to the three sensors.

TABLE II

PSD AND FREQUenCy VAlues (FIG. 15)

\begin{tabular}{cccc}
\hline \hline Sensor & $\begin{array}{c}\text { Attenuation } \\
(\mathrm{dB})\end{array}$ & $\begin{array}{c}\text { PSD } \\
(\mathrm{dB} / \mathrm{Hz})\end{array}$ & $\begin{array}{c}\text { Frequency } \\
(\mathrm{kHz})\end{array}$ \\
\hline \multirow{2}{*}{ MFC-P1 } & 3 & -92.55 & 163.8 \\
& 10 & -99.55 & 333.2 \\
MFC-P2 & 3 & -107 & 176.1 \\
& 10 & -114 & 364 \\
\multirow{2}{*}{ R15I-AST } & 3 & -78.43 & 245.6 \\
& 10 & -85.43 & 359.6 \\
\hline \hline
\end{tabular}

Two points in the PSD curves were highlighted to compare the signals as shown in Table II. The results show that the conventional sensor was more sensitivity than the MFC-P1 and MFC-P2 sensor.

For the analyzed points, the 3-dB attenuation for the R15I sensor was at frequency of $245.6 \mathrm{kHz}$ and represents a higher band than both sensors MFC-P1 and MFC-P2, which were attenuated at frequencies of $163.8 \mathrm{kHz}$ and $176.1 \mathrm{kHz}$, respectively. The $10-\mathrm{dB}$ attenuation for the conventional sensor was at frequency of $359.6 \mathrm{kHz}$ and represents a higher band only than the MFC-P1 sensor, which was attenuated at the $333.2 \mathrm{kHz}$. The wider band for this attenuation was obtained for the MFC-P2, although this sensor was less sensitivity for PDs. However, the conventional sensor was more sensitivity due to higher gain, as shown in Fig. 5, but the MFC sensors have a near range of the R15I-AST sensor.

Therefore, the results show that the discharge spectrum is more significant up to $250 \mathrm{kHz}$ and has high attenuation at $400 \mathrm{kHz}$. The PSD analysis show that the MFC sensors are feasible for acoustic PD measurement in power transformers due to high similarity of the results compared to the conventional sensor in the response of the discharge signals.

Finally, it is important to note that the relative low sensitivity observed for the MFC sensors compared to the conventional AE sensor can be improved by using an appropriate amplifier.

\section{Conclusions}

The purpose of this study was to analyze the feasibility of MFC sensors for measuring acoustic signals from partial discharges in power transformers. Although the experimental setup has used a full discharge to provide high signal-to-noise 
ratio measurements, all tests were carried out under the same conditions for an effective comparison of the MFC sensors with a conventional sensor (RS15I) commonly used for localization of partial discharges.

The results obtained by the two types of MFC sensors are similar to the results obtained using a conventional sensor, indicating the feasibility of these sensors for the measurement of acoustic signals from partial discharge events. This inference é based on the energy, RMS and Akaike criteria, which indicated good sensitivity of the MFC sensors to detect acoustic emission waves, besides the high correlation between the results obtained by the MFC sensors and those obtained by the conventional sensor. Although the relative sensitivity of the MFC sensors is lower compared to the conventional sensor, it can be increased by the use of an appropriate amplifier. In addition, MFC sensors are low-profile, low-cost and flexible compared to the conventional sensors and are readily available, making the use of these devices particularly attractive in large power transformers multiple sensors may be required for the reliable localization of partial discharges.

Finally, as a suggestion of future research, multiple MFC sensors can be installed in a large transformer for the effective detection and localization of real partial discharges by combining the electromagnetic and acoustic emission methods.

\section{ACKNOWLEDGMENT}

The authors are grateful to the anonymous reviewers for their valuable recommendations for improving this article.

\section{REFERENCES}

[1] C. Zachariades, R. Shuttleworth, R. Giussani, and R. MacKinlay, "Optimization of a high-frequency current transformer sensor for partial discharge detection using finite-element analysis," IEEE Sensors J., vol. 16, no. 20, pp. 7526-7533, Oct. 2016. [Online]. Available: http://dx.doi.org/10.1109/JSEN.2016.2600272

[2] M. Shafiq, L. Kutt, M. Lehtonen, T. Nieminen, and M. Hashmi, "Parameters identification and modeling of high-frequency current transducer for partial discharge measurements," IEEE Sensors J., vol. 13, no. 3, pp. 1081-1091, Mar. 2013. [Online]. Available: http://dx.doi.org/10.1109/JSEN.2012.2227712

[3] B. Castro et al., "Partial discharge monitoring in power transformers using low-cost piezoelectric sensors," Sensors, vol. 16, no. 8, p. 1266, Aug. 2016. [Online]. Available: http://dx.doi.org/10.3390/s16081266

[4] S. Biswas, C. Koley, B. Chatterjee, and S. Chakravorti, "A methodology for identification and localization of partial discharge sources using optical sensors," IEEE Trans. Dielectr. Electr. Insul., vol. 19 no. 1, pp. 18-28, Feb. 2012. [Online]. Available: http://dx.doi. org/10.1109/TDEI.2012.6148498

[5] B. A. Castro, G. A. M. Clerice, A. L. Andreoli, F. S. de Campos, and J. A. C. Ulson, "A low cost system for acoustic monitoring of partial discharge in power transformer by piezeletic sensor," IEEE Latin Amer. Trans., vol. 14, no. 7, pp. 3225-3231, Jul. 2016. [Online]. Available: http://dx.doi.org/10.1109/TLA.2016.7587624

[6] S. A. Boggs, "Partial discharge: Overview and signal generation," IEEE Elect. Insul. Mag., vol. 6, no. 4, pp. 33-39, Jul. 1990. [Online]. Available: http://dx.doi.org/10.1109/57.63057

[7] I. N. da Silva et al., Eds., "Intelligent systems for the detection of internal faults in power transmission transformers," in Advances in Expert Systems. Rijeka, Croatia: InTech, 2012, pp. 3-27. [Online]. Available: http://dx.doi.org/10.5772/51417

[8] B. Danouj, S. A. Tahan, and E. David, "Using a new generation of piezoelectric sensors for partial discharge detection," Measurement, vol. 46, no. 1, pp. 660-666, Jan. 2013. [Online]. Available: http://dx.doi. org/10.1016/j.measurement.2012.09.005
[9] High-Voltage Test Techniques-Partial Discharge Measurements, 2nd ed., IEC Standard 60270, IEC Publication, 2000.

[10] F. Álvarez, F. Garnacho, J. Ortego, and M. Á. Sánchez-Urán, “Application of HFCT and UHF sensors in On-line partial discharge measurements for insulation diagnosis of high voltage equipment," Sensors, vol. 15, no. 4, pp. 7360-7387, Apr. 2015. [Online]. Available: http:// dx.doi.org/10.3390/s150407360

[11] D.-J. Kweon, S.-B. Chin, H.-R. Kwak, J.-C. Kim, and K.-B. Song, "The analysis of ultrasonic signals by partial discharge and noise from the transformer," IEEE Trans. Power Del., vol. 20, no. 3, pp. 1976-1983, Jul. 2005. [Online]. Available: http://dx.doi.org/10. 1109/TPWRD.2004.833923

[12] M. Siegel, M. Beltle, S. Tenbohlen, and S. Coenen, "Application of UHF sensors for PD measurement at power transformers," IEEE Trans. Dielectr. Electr. Insul., vol. 24, no. 1, pp. 331-339, Feb. 2017. [Online]. Available: http://dx.doi.org/10.1109/TDEI.2016.005913

[13] S. M. Markalous, S. Tenbohlen, and K. Feser, "Detection and location of partial discharges in power transformers using acoustic and electromagnetic signals," IEEE Trans. Dielectr. Electr. Insul., vol. 15, no. 6, pp. 1576-1583, Dec. 2008. [Online]. Available: http://dx.doi. org/10.1109/TDEI.2008.4712660

[14] A. Kraetge, S. Hoek, M. Koch, and W. Koltunowicz, "Robust measurement, monitoring and analysis of partial discharges in transformers and other HV apparatus," IEEE Trans. Dielectr. Electr. Insul., vol. 20, no. 6, pp. 2043-2051, Dec. 2013. [Online]. Available: http://dx.doi. org/10.1109/TDEI.2013.6678852

[15] J. Fuhr and T. Aschwanden, "Identification and localization of PD-sources in power-transformers and power-generators," IEEE Trans. Dielectr. Electr. Insul., vol. 24, no. 1, pp. 17-30, Feb. 2017. [Online]. Available: http://dx.doi.org/10.1109/TDEI.2016.005951

[16] M. Siegel, S. Tenbohlen, S. Coenen, B. Dolata, G. Luna, and S. Louise, "Practical sensitivity of on line UHF PD monitoring on large power transformers," in Proc. Cigre SC A2 Colloq., Shangai, China, 2015, pp. 1-12. [Online]. Available: https://www.researchgate. net/publication/283302891_Practical_Sensitivity_of_online_UHF_PD_ Monitoring_on_Large_Power_Transformers

[17] W. G. Drossel, S. Hensel, and M. Nestler, "Evaluation of actuator, sensor, and fatigue performance of piezo-metal-composites," IEEE Sensors J., vol. 14, no. 7, pp. 2129-2137, Jul. 2014. [Online]. Available: http://dx. doi.org/10.1109/JSEN.2013.2296143

[18] A. Giuliano and M. Zhu, "A passive impedance matching interface using a PC permalloy coil for practically enhanced piezoelectric energy harvester performance at low frequency," IEEE Sensors J., vol. 14, no. 8, pp. 2773-2781, Aug. 2014. [Online]. Available: http://dx.doi. org/10.1109/JSEN.2014.2316091

[19] F. H. Krueger, Partial Discharge Detection in High-Voltage Equipment, 1st ed. London, U.K.: Butterworths, 1989.

[20] R. Sarathi, P. D. Singh, and M. G. Danikas, "Characterization of partial discharges in transformer oil insulation under AC and DC voltage using acoustic emission technique," J. Elect. Eng., vol. 58, no. 2, pp. 91-97, Mar. 2007. [Online]. Available: http://iris.elf.stuba.sk/JEEEC/ data/pdf/2_107-05.pdf

[21] L. E. Lundgaard, "Partial discharge-Part XIV: Acoustic partial discharge detection-practical application," IEEE Elect. Insul. Mag., vol. 8, no. 5, pp. 34-43, Sep./Oct. 1992. [Online]. Available: http://dx. doi.org/10.1109/57.156943

[22] G. C. Stone, "Partial discharge diagnostics and electrical equipment insulation condition assessment," IEEE Trans. Dielectr. Electr. Insul., vol. 12, no. 5, pp. 891-904, Oct. 2005. [Online]. Available: http://dx.doi.org/ 10.1109/TDEI.2005.1522184

[23] B. T. Phung, T. R. Blackburn, and Z. Liu, "Acoustic measurements of partial discharge signals," J. Elect. Electron. Eng., vol. 21, no. 1, pp. 41-47, 2001.

[24] A. H. Meitzler et al., IEEE Standard on Piezoelectricity: An American National Standard, IEEE-ANSI Standard 176, New York, NY, USA, 1988, p. 66. [Online]. Available: http://dx.doi.org/10.1109/ IEEESTD.1988.79638

[25] Physical Acoustics-MISTRAS Group. (2016). R15I-AST SensorIntegral Preamplifier Sensor. Product Data Sheet. Accessed on Aug. 18, 2016. [Online]. Available: http://www.physicalacoustics.com/ content/literature/sensors/Model_R15I-AST.pdf

[26] S. Sharab and A. Erturk, "Coupling of experimentally validated electroelastic dynamics and mixing rules formulation for macro-fiber composite piezoelectric structures," J. Intell. Mater. Syst. Struct., vol. 28, no. 12, pp. 1575-1588, 2016. [Online]. Available: http://dx.doi. org/10.1177/1045389X16672732 
[27] H. A. Tinoco and D. J. Marulanda, "Damage identification in active plates with indices based on Gaussian confidence ellipses obtained of the electromechanical admittance," J. Nondestruct. Eval., vol. 34, p. 28, Sep. 2015. [Online]. Available: http://dx.doi.org/10.1007/s10921-0150299-3

[28] E. S. da Freitas and F. G. Baptista, "Experimental analysis of the feasibility of low-cost piezoelectric diaphragms in impedance-based SHM applications," Sens. Actuators A, Phys., vol. 238, pp. 220-228, Feb. 2016. [Online]. Available: http://dx.doi.org/10.1016/j.sna.2015.11.031

[29] R. Liang and Q.-M. Wang, "High sensitivity piezoelectric sensors using flexible PZT thick-film for shock tube pressure testing," Sens. Actuators A, Phys., vol. 235, pp. 317-327, Nov. 2015. [Online]. Available: http://dx.doi.org/10.1016/j.sna.2015.09.027

[30] V. A. D. de Almeida, F. G. Baptista, and P. R. de Aguiar, "Piezoelectric transducers assessed by the pencil lead break for impedance-based structural health monitoring," IEEE Sensors J., vol. 15 , no. 2 , pp. $693-702$, Feb. 2015. [Online]. Available: http://dx. doi.org/10.1109/JSEN.2014.2352171

[31] J. Ilg, S. J. Rupitsch, and R. Lerch, "Impedance-based temperature sensing with piezoceramic devices," IEEE Sensors J., vol. 13, no. 6, pp. 2442-2449, Jun. 2013. [Online]. Available: http://dx.doi.org/ 10.1109/JSEN.2013.2256121

[32] F. G. Baptista, J. V. Filho, and D. J. Inman, "Sizing PZT transducers in impedance-based structural health monitoring," IEEE Sensors J., vol. 11, no. 6, pp. 1405-1414, Jun. 2011. [Online]. Available: http://dx.doi.org/ 10.1109/JSEN.2010.2098865

[33] F. G. Baptista, D. E. Budoya, V. A. D. de Almeida, and J. A. C. Ulson, "An experimental study on the effect of temperature on piezoelectric sensors for impedance-based structural health monitoring," Sensors, vol. 14, no. 1, pp. 1208-1227, Nov. 2013. [Online]. Available: http:// dx.doi.org/10.3390/s140101208

[34] T. Boczar, S. Borucki, A. Cichon, and D. Zmarzly, "Application possibilities of artificial neural networks for recognizing partial discharges measured by the acoustic emission method," IEEE Trans. Dielectr Electr. Insul., vol. 16, no. 1, pp. 214-223, Feb. 2009. [Online]. Available: http://dx.doi.org/10.1109/TDEI.2009.4784570

[35] G. Robles, J. M. Fresno, and J. M. M. Tarifa, "Separation of radiofrequency sources and localization of partial discharges in noisy environments," Sensors, vol. 15, no. 5, pp. 9882-9898, 2015. [Online]. Available: http://dx.doi.org/10.3390/s150509882

[36] P. R. de Aguiar, E. C. Bianchi, and R. C. Canarim, "Monitoring of grinding burn by acoustic emission," in Acoustic Emission, W. Sikorski, Ed. Rijeka, Croatia: InTech, 2012, pp. 341-364. [Online]. Available: http:/ dx.doi.org/10.5772/31339

[37] M. Yuan, Z. Pang, S. Dong, and S. Wang, "Research to real-time algorithm of crack initiation monitoring based on Welch's method," in Proc. IEEE Chin. Guid., Navigat. Control Conf., Yantai, China, Aug. 2014, pp. 2401-2406. [Online]. Available: https://doi.org/10.1109/ CGNCC.2014.7007545

[38] M. N. Bawane and K. M. Bhurchandi, "Classification of mental task based on EEG processing using self organising feature map," in Proc. 6th Int. Conf. Intell. Hum.-Mach. Syst. Cybern., Hangzhou, China, Aug. 2014, pp. 240-244. [Online]. Available: https://doi. org/10.1109/IHMSC.2014.160

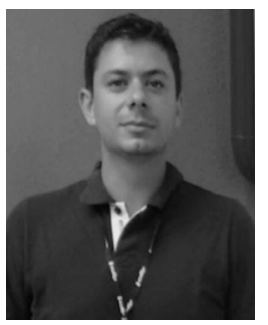

Bruno Albuquerque de Castro (S'08-M'16) received the B.S. and M.Sc. degrees in electrical engineering from Sao Paulo State University, Bauru, Brazil, in 2012 and 2016, respectively, where he is currently pursuing the Ph.D. degree in electrical engineering with scholarship provided by São Paulo Research Foundation. His research field focuses are in the structural health monitoring, failures diagnostics at the civil, mechanic structural, intelligent systems, data acquisition, electronic instrumentation, acoustic emission, piezoelectricity, and instrumenta-

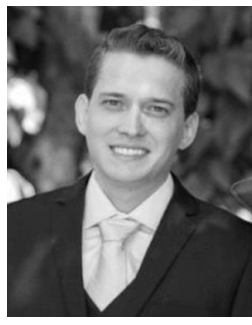

Danilo de Melo Brunini (M'16) received the B.S. degree in control and automation engineering from Sao Paulo State University, Sorocaba, Brazil, in 2012, and the M.Sc. degrees in electrical engineering from Sao Paulo State University, Brazil, in 2017. He has about 6 years of experience in industrial automation projects working at Emerson Process Brasil from 2011 to 2015 and with Andritz Feed and Biofuel Brazil from 2015 to 2016. His field of research focuses on intelligent systems, acoustic emission, and electrical power systems.

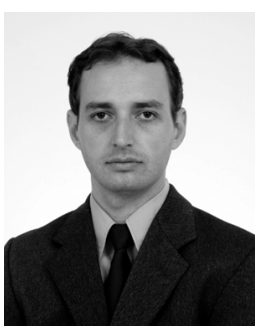

Fabricio Guimarães Baptista (S'08-M'11) received the B.S. and $\mathrm{Ph} . \mathrm{D}$. degrees in electrical engineering from Sao Paulo State University, Ilha Solteira, Brazil, in 2006 and 2010, respectively. From 2010 to 2011, he was a Visiting Scholar with the Center for Intelligent Material Systems and Structures, Virginia Tech, Blacksburg, VA, performing research in the field of structural health monitoring and piezoelectric transducers. $\mathrm{He}$ is currently an Assistant Professor with the Department of Electrical Engineering, Sao Paulo State University, Bauru, where he coordinates the Laboratory of Transducers and Data Acquisition, working in the field of piezoelectricity, structural health monitoring, signal processing, data acquisition, and electronic instrumentation.

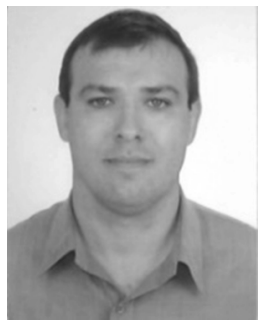

André Luiz Andreoli (M'15) received the degree in electrical engineering from Sao Paulo State University, Bauru, Brazil, in 1997, the master's degree in industrial engineering from Sao Paulo State University in 2005, and the Ph.D. degree in electrical engineering from Escola de Engenharia de São Carlos in 2011. He is currently a Professor with the Department of Electrical Engineering, Faculty of Engineering, São Paulo State University, Bauru. His field of research focuses in industrial electronics, power quality, electrical machines and drives.

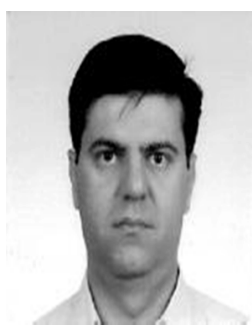

José Alfredo Covolan Ulson was born in Bauru in 1966. He received the electrical engineering degree from the Sao Paulo State University Bauru Campus in 1991, and the master's and Ph.D. degree from Sao Paulo State University, in 1997 and 2002, respectively. $\mathrm{He}$ is currently an Assistant Professor with the Department of Electrical Engineering. His field of research focuses in the areas of intelligent systems and identification systems applied to process control and electrical power systems. 